\title{
A Method to Divide Flow Units of Carbonate Reservoir
}

\author{
Li Jianxin ${ }^{1, a}$, Li Kongchou, ${ }^{1, b}$, Wang Shuqin ${ }^{1, c}$ and Luo Man ${ }^{1, d}$ \\ ${ }^{1}$ Research Institute of Petroleum Exploration and Development, CNPC, BeiJing \\ alijianxin1@petrochina.com.cn, ${ }^{b}$ likongchou@petrochina.com.cn, cwshuqin@petrochina.com.cn, \\ duoman@petrochina.com.cn
}

Keywords: Carbonate oilfield, reservoir type; reservoir flow unit, neural network clustering technology, geological model

Abstract. Oil reservoir $\Gamma$ north of Zhanzhol, a fracture-pore typed carbonate oilfield, has a variety of reservoir types. According to the combination pattern of different pores and the relationship between porosity and permeability, carbonate reservoirs are classified into four types which are fracture-cavity typed, fracture-pore typed, pore typed and fracture typed, and the identification of which by well logging data is realized. In order to appraise the producing degree of reservoirs more exactly, key parameters affecting the producing degree of fracture-pore typed and pore typed reservoirs are selected separately, taking which as clustering variants, with the consideration of both matrix and fracture, a flow unit division method for carbonate reservoir is established, four types of reservoirs are further divided into six kinds of reservoir flow units by the means of neural network clustering technology, and a three dimensional geological model is built to describe the distribution of reservoir flow units.

\section{Introduction}

Carbonate reservoir, with a strong heterogeneity, has a variety of reservoir spaces and combination types ${ }^{[1]}$.In this paper, the concept of reservoir flow unit refers to the basic reservoir unit with consistent geological, petrological, and hydrodynamic features within a given oil and gas reservoirs that is different from other rocks ${ }^{[2,3]}$.

\section{Overview}

Zhanzhol Oilfield, located in Zhaerkameisi Uplift zone in the eastern margin of the Pre-Caspian Basin, is a large carbonate oilfield with condensate gas cap and edge - bottom water. The major oil reservoir, $\Gamma$ north can be divided into ten subzones. The reservoir, composed of grain limestone, belongs to open platform facies deposition, with reservoir space of intergranular (dissolved) pore, body cavity pore and intragranular pore and fracture of micro-fracture and minor dissolved cavity. Reservoir porosity is $6.0 \%-20.73 \%$, with $9.70 \%$ in average, and permeability is $0.05-518.31 \times 10^{-3} \mu \mathrm{m}^{2}$, with $28.45 \times 10^{-3} \mu \mathrm{m}^{2}$ in average.

\section{Division and Identification of Reservoir Type}

Reservoir Type Classification. According to the combination patterns of different types of pores, carbonate reservoir can be divided into seven types by means of triangular diagram taxonomy ${ }^{[4]}$ (Fig. 1). According to the special area that different types of reservoir core samples locate in the porosity-permeability cross plot, reservoirs of $\Gamma$ north are reduced into four types, which are 
fracture-cavity typed, fracture-pore typed, pore typed and fracture typed, in order to identify them using conventional well logging data easily (Fig. 2).

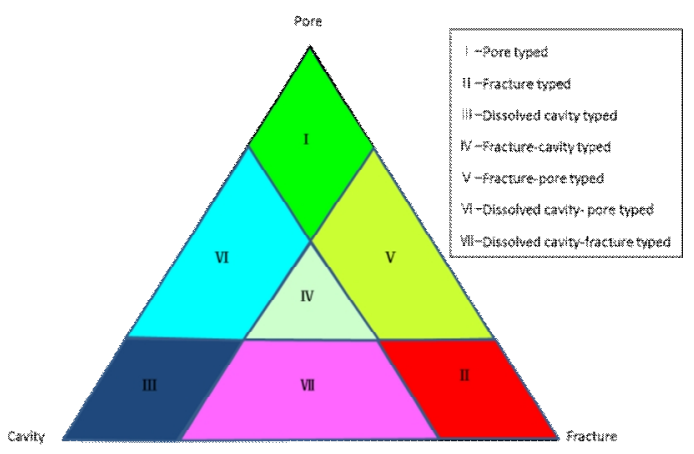

Fig.1 Pore type classification triangle diagram of carbonate reservoir

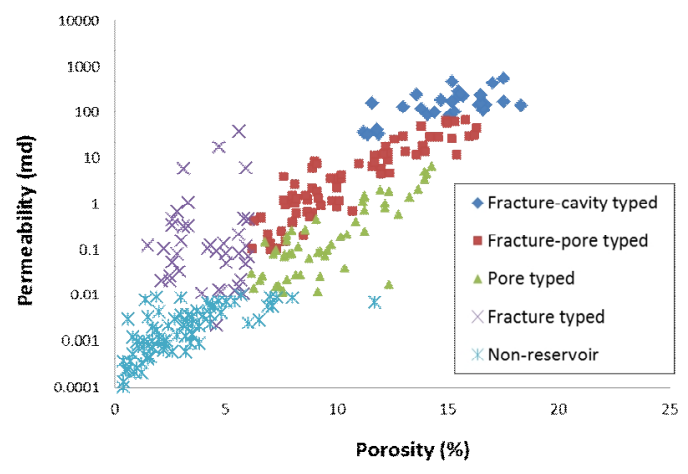

Fig.2 Cross-plot of core porosity-permeability

Well Logging Identification of Different Reservoir Types. Different types of carbonate reservoirs have different pore space strcutres and well logging response features ${ }^{[5]}$. Based on core analysis data and reservoir type division results, the well logging response features of different types of reservoirs in oil reservoir $\Gamma$ north are summerized as in Table 1.

Table 1 Statistics of Well logging Responce of Different Types of Reservoirs

\begin{tabular}{ccccccccc}
\hline Reservoir type & $\begin{array}{c}\text { GR } \\
(\mathrm{API})\end{array}$ & $\begin{array}{c}\text { LLD } \\
(\Omega \cdot \mathrm{m})\end{array}$ & $\begin{array}{c}\text { LLS } \\
(\Omega \cdot \mathrm{m})\end{array}$ & $\begin{array}{c}\text { CNL } \\
(\%)\end{array}$ & $\begin{array}{c}\text { Denstiy } \\
\left(\mathrm{g} / \mathrm{cm}^{3}\right)\end{array}$ & $\begin{array}{c}\text { DT } \\
(\mu \mathrm{s} / \mathrm{m})\end{array}$ & $\begin{array}{c}\text { Porosity } \\
(\%)\end{array}$ & $\begin{array}{c}\text { PE } \\
(\mathrm{b} / \mathrm{e})\end{array}$ \\
\hline \multirow{2}{*}{ Fracture-cavity } & $<30$ & $50-1000$ & $10-100$ & $\geq 10$ & $\leq 2.54$ & $\geq 199$ & $12-30$ & $5.0 \pm$ \\
\hline \multirow{2}{*}{ Fracture-pore } & $<30$ & $10-500$ & $10-500$ & $6-15$ & $2.61-2.46$ & $182-212$ & $6-15$ & $5.0 \pm$ \\
\hline \multirow{2}{*}{ Pore } & $<20$ & $30-500$ & $30-500$ & $6-3$ & $2.35-2.65$ & $182-208$ & $6-15$ & 5.08 \\
\hline \multirow{2}{*}{ Fracture } & $10-70$ & $5-200$ & $5-201$ & $<6$ & $2.65-2.70$ & $165-175$ & $<6$ & $3.14-5.08$ \\
\hline
\end{tabular}

Fracture-cavity reservoir is characterized by low GR value, high photoelectric trapping index, obvious amplitude difference of three resistivity curves (LLD, LLS and microspherical resistivity), high value of three porosity curves ( bulk density, compensated neutron and transit time interval) (Fig. 3a). Fracture-pore type reservoir is characterized by low GR value, high photoelectric trapping index, medium-low LLD value, smaller amplitude difference of three resistivity curves than that of fracture-cavity reservoir, medium-high value of three porosity curves and medium-low porosity value, smaller than that of fracture-cavity reservoir (Fig. 3b). Pore type reservoir is characterized by low GR value, high photoelectric trapping index, small amplitude difference or coincidence of three resistivity curves , medium-low LLD value, medium-low and coincidece of neutron curve and density curve values, medium-low transit time interval value and porosity value closed to that of fracture-pore type reservoir (Fig. 3c). Fracture type reservoir is characterized by low GR value, medium-high photoelectric trapping index, low LLD value, sawtooth-shaped variation of microspherical resistivity curve, higher than LLD value sometimes, basically coinciding of three porosity curve values and low porosity value (Fig. 3d). 


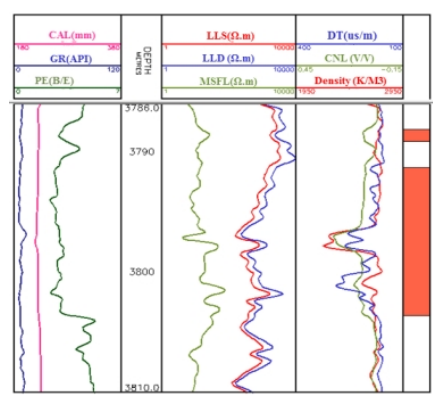

Fig. 3a Fra.-cavity typed

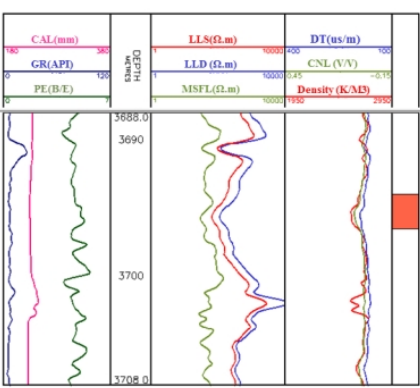

Fig. 3b Frac.-pore typed

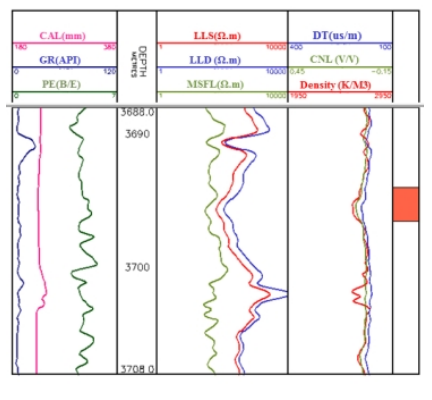

Fig. 3c Pore typed

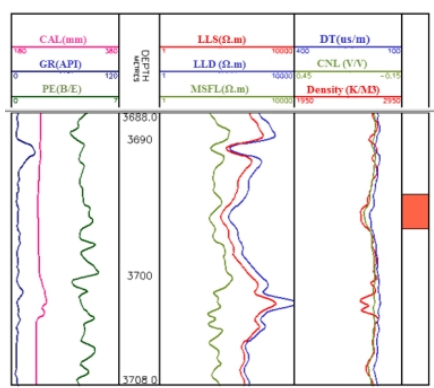

Fig. 3d Fra. typed

Fig. 3 Conventional Logging Response Characteristics of Different Types of Reservoirs

\section{Flow unit division and characterization based on reservoir type}

Flow Unit Division Background. Fluid production intensity of single layer is calculated using the fluid production profile data of 122 oil wells of $\Gamma$ north, and the reservoirs can be divided into four types: reservoirs with good producing degree, whose fluid production intensity is greater than or equal to $2 \mathrm{~m}^{3} /(\mathrm{d} . \mathrm{m})$; reservoirs with fair producing degree, whose fluid production intensity is $0.5-2.0$ $\mathrm{m}^{3} /($ d.m); reservoirs with poor producing degree, whose fluid production intensity is less than 0.5 $\mathrm{m}^{3} /($ d.m); reservoirs with no producing degree, which has no fluid production.

Fracture-cavity typed reservoirs have large fluid production intensity, high and concentrated producing degree, reservoirs with good and fair producing degree accounting for $94.1 \%$. Fracture-pore typed reservoirs have high producing degree, reservoirs with good and fair producing degree accounting for $83.3 \%$, but reservoirs with poor and no producing degree also accounting for $16.7 \%$. Pore typed reservoirs have similar proportion of reservoirs with different producing degrees, reservoirs with good, fair, poor and no producing degree accounting for 25.7\%, 32.0\%, $12.9 \%$ and $29.4 \%$ respectively. Fracture typed reservoir is few, whose liquid production profile shows no production. Pore typed and fracture-pore typed reservoirs account large total thickness and significantly different producing degree. For the purpose of evaluating reservoir producing degree more accurately, it is necessary to subdivide reservoir flow unit based on reservoir types ${ }^{[6,7]}$.

Flow Unit Division and Characterization. On the basis of core analysis data, the geological parameters characterizing reservoir flow features are calculated applying well logging interpretation parameters, then, taking producing degree as discriminating parameters, the geological parameters affecting reservoir producing degree are selected as clustering variables, finally, by the application of neural network clustering technology, the reservoir flow unit of individual well is divided ${ }^{[8]}$ and a 3D geological models of reservoir flow units are built in order to characterize the distribution of reservoir flow units.

Selection of Clustering Variables. Various geological parameters calculated by well logging parameters are used to draw cross plot to make correlation analysis with producing degree of reservoirs. For fracture-pore typed reservoirs, four geological parameters significantly affecting producing degree are selected as clustering variables, which are reservoir quality index (RQI), pore throat radius at mercury saturation of $50 \%\left(R_{50}\right)$, the ratio of fracture permeability to matrix permeability $\left(K_{\mathrm{f}} / K_{\mathrm{m}}\right)$ and total permeability $\left(K_{\mathrm{f}}+K_{\mathrm{m}}\right)$. For pore typed reservoirs, three geological parameters are selected, which are $R Q I, R_{50}$ and $K_{\mathrm{m}}$. Reservoir quality index (RQI) is calculated as Eq. $1^{[1]}$ :

$$
R Q I=0.0314 \sqrt{K / \phi_{\mathrm{e}}}
$$


where, $R Q I$-reservoir quality index, $\mu \mathrm{m}$; K-- reservoir absolute permeability, $\mathrm{mD}$; $\varphi_{\mathrm{e}}$-reservoir effective porosity, $\mathrm{f}$.

Taking Winland formula ${ }^{[2]}$ as reference, the relationship of throat radius at mercury saturation of $50 \%$ to absolute permeability and porosity is established Eq. 2, with a correlation coefficient is 0.951 :

$$
\lg R_{50}=-0.0481+0.014 \varphi_{\mathrm{e}}+0.3951 \lg K
$$

For fracture-pore typed reservoir, the cross plot between $R Q I, R_{50}, K_{\mathrm{f}} / K_{\mathrm{m}}, K_{\mathrm{f}}+K_{\mathrm{m}}$ and producing degree shows (Fig. 4) the producing degree positively correlates with $R Q I, R_{50}$ and $K_{\mathrm{f}}+K_{\mathrm{m}}$ and correlates with $K_{\mathrm{f}} / K_{\mathrm{m}}$ to some extent. For pore typed reservoir, the cross plot between $R Q I, R_{50}, K_{\mathrm{m}}$ and producing degree shows (Fig. 5) that the producing degree positively correlates with $R Q I, R_{50}$ and $K_{\mathrm{m}}$. Whether fracture-pore typed or pore typed reservoirs, the data points of different producing degrees are partially overlapping, resulting in that the producing degree of reservoirs can't be accurately characterized by one separate geological parameter, therefore, cluster analysis technology is adopted to divide reservoir flow units, which will consider multiple factors.
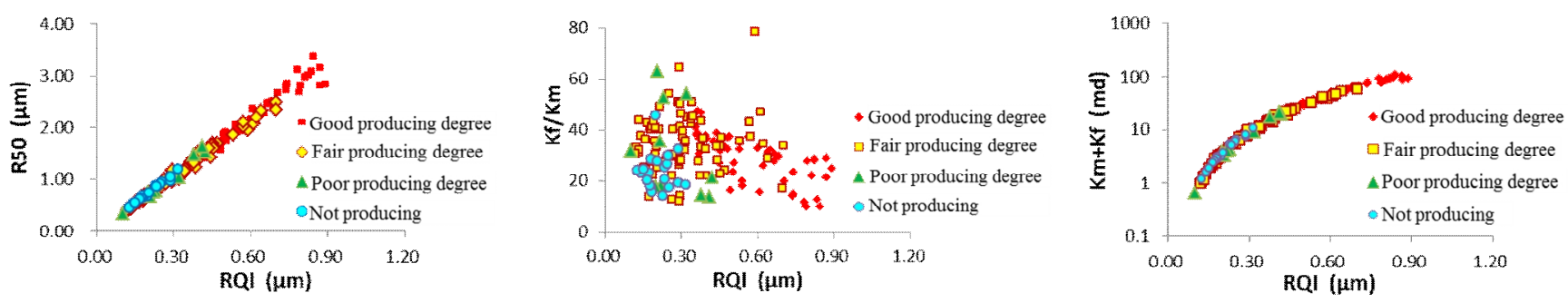

Fig. 4Cross plot of Different Geological Parameters and Reservoir Producing Degrees for Fra.-Pore Typed Reservoirs
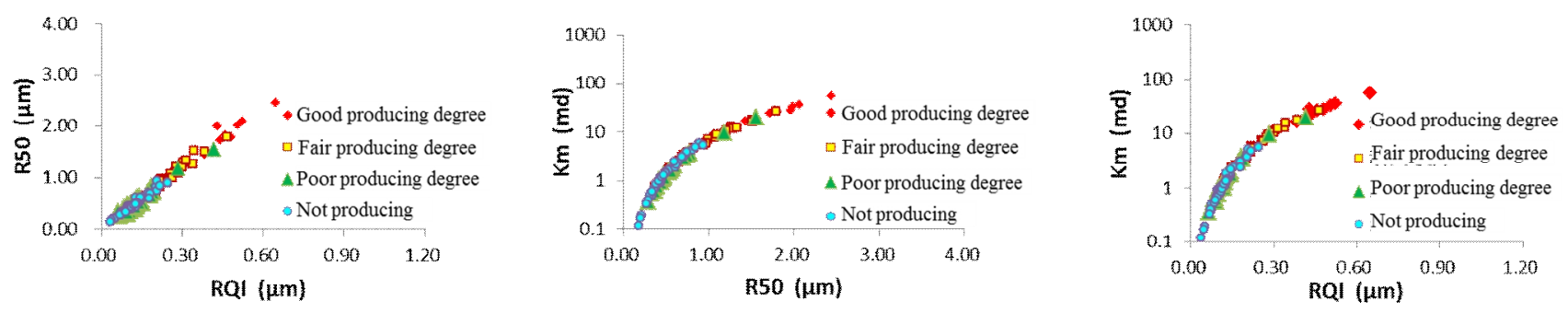

Fig.5Cross plot of Different Geological Parameters and Reservoir Producing Degrees for Pore Typed Reservoirs

Division and Characterization of Flow Units. The flow unit division of oil reservoir $\Gamma$ north follows such principles: the fracture-cavity typed reservoir and the fractured reservoirs are taken as one type of flow unit, represented letters A and D respectively. For fracture-pore typed and pore typed reservoirs with significantly different producing degrees and large proportion in total reservoir thickness, with the selected geological parameters as clustering variables, neural network technology are applied to perform clustering analysis, by which they are subdivided into two types of flow units, represented by letters $\mathrm{B} 1, \mathrm{~B} 2$ and $\mathrm{C} 1, \mathrm{C} 2$ respectively.

According to the reservoir flow unit division results of individual wells, a 3D geological model of flow units of oil reservoir $\Gamma$ north is built adopting sequential indicator simulation method to characterize the distribution of different flow units. Different flow units have different distribution area and location on the plane as well as different distribution location and probability in vertically. 


\section{Appraisal of Flow Unit}

Different type of reservoir flow units possess different relative permeability curves(Fig. 6), and the same kind of flow units presents similar producing degrees(Fig. 7). Flow unit A presents good producing degree and is defined as high level flow unit, flow unit B1 and $\mathrm{C} 1$ present fair producing degree and are defined as fair level flow unit, flow unit $\mathrm{C} 2$ and D present poor producing degree and are defined as poor level flow unit.

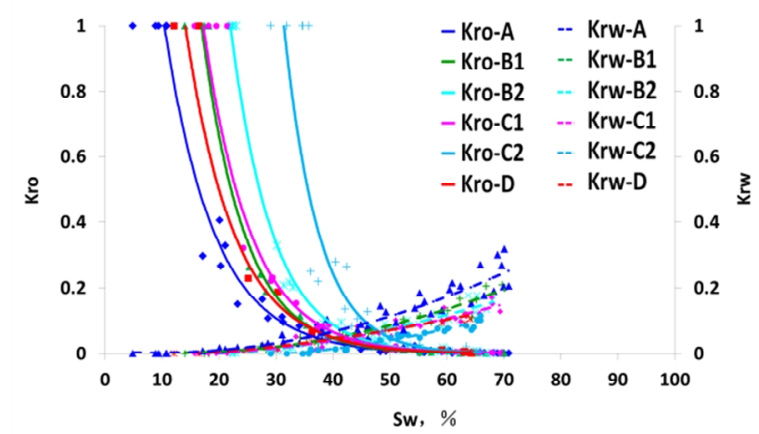

Fig. 6 Relative Permeability Curves of Different Kinds of Flow Units

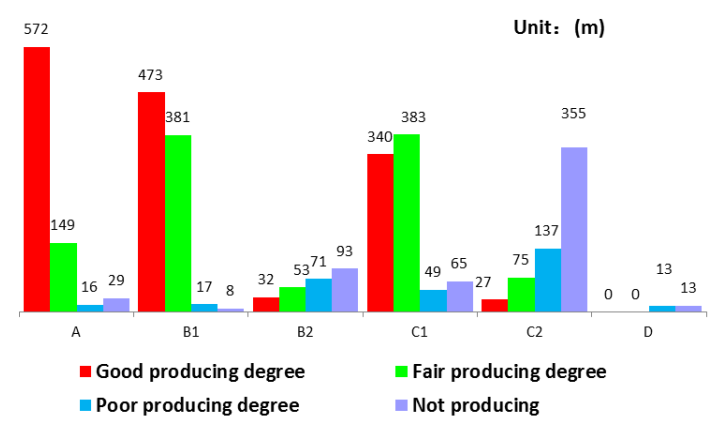

Fig. 7 Thickness of Each Kind of Flow Unit with Different Producing degree

\section{Conclusion}

With the division of four types of reservoirs of oil reservoir $\Gamma$ north, which are fracture-cavity typed, fracture-pore typed, pore typed and fracture typed, the seemingly confused relationship between porosity and permeability present same regularity, however, the producing degree of fracture-pore typed and pore typed reservoirs each varies in large degree. After the subdivision of four types of reservoir into six kind of flow units, the same kind of flow unit possesses similar relative permeability curves, and presents similar producing degrees.

\section{References}

[1] Lucia F J. Carbonate reservoir characterization[M]. Beijing: Petroleum Industry Press, 2011: 16-22.

[2] Jin Qinghuan, Zhang Li, Wei Kai et al. Bioherm petroleum reservoir types and features in main sedimentary basins of the South China Sea [J]. Journal of Earth Science, 2012, 23 (6): 828-841

[3] Amaefule J O, Altunbay M, Tiab D, et al. Enhanced reservoir description: Using core and log data to identify hydraulic(flow) units and predict permeability in uncored intervals/wells[R]. SPE 26436, 1993.

[4] Qiang Zitong. Carbonate reservoir geology[M]. Dongying: China University of Petroleum Press, 2007: 52-95.

[5] Yong Shihe, Zhang Chaomo. Log data processing and comprehensive interpretation[M]. Dongying: China University of Petroleum Press, 1996: 289-298.

[6] Liu Jiyu. Advances in the study of reservoir flow unit[J]. Advances in Earth Science, 2000, 15(3): 303-306.

[7] Wang Zhizhang, He Gang. The classification method and application of reservoir flow units[J]. Natural Gas Geoscience, 2010, 21(3): 362-366.

[8] Sun Zhixue, Yao Jun, Sun Zhilei, et al. The application of clustering analysis based on neural network technology in reservoir flow unit classification[J]. Geophysical \& Geochemical Exploration, 2011, 35(3): 249-353. 